\title{
System Dynamics Analysis of Integrated Pipeline Corridor Based on Risk Loops
}

\author{
Weixin FENG*, Dan CHANG, Daqing GONG, Jiren SHEN
}

\begin{abstract}
With the continuous improvement of China's infrastructure, integrated pipeline corridors have become an indispensable part of intelligent city management. Because of complex environments and numerous potential failures in integrated pipeline corridor operation and maintenance, the risks in integrated pipeline corridors present dynamic characteristics. The analysis of only their static characteristics is therefore insufficient. In this paper, based on the combination of the design structure matrix (DSM) method and the principle of pairwise comparison in the analytic hierarchy process (AHP), we determine the interaction and the strength of the relationship between the risks of integrated pipeline corridor operation and maintenance, construct a dynamic risk model based on system dynamics, and analyze the influence mechanism of the risk control input on the system risk in integrated pipeline corridor operation and maintenance management. The simulation results show that the integrated pipeline corridor operation and maintenance risks form various risk paths under the influence of unreasonable design and construction, third-party damage, natural disasters and management team level factors. The risk control input can reduce the integrated pipeline corridor operation and maintenance risk to a certain extent.
\end{abstract}

Keywords: integrated pipeline corridor; operation and maintenance management; risk study; system dynamics

\section{INTRODUCTION}

As a public tunnel for the centralized laying of municipal utility pipelines, integrated pipeline corridors can effectively solve many urban problems such as repeated road excavation and environmental deterioration caused by traditional pipeline laying and greatly improve the urban living environment. Integrated pipeline corridors also enhance the comprehensive carrying capacity of the city, which is a key aspect of the sustainable development of municipalities and their infrastructure [1]. China's integrated pipeline corridor construction has been rapidly developed after 2015 with the support of a series of government policies. It is now in the stage of promotion and construction, and the construction scale and construction level have been in the leading position in the world [2]. However, due to the increased concentration of various types of high-risk pipelines in central corridors and the resulting elevated risk of cable breakage and pipeline leakage, disasters such as poisoning, explosion, and fire can easily occur under the joint action of internal and external factors and the mutual influence between pipelines [3]. After the construction phase of integrated pipeline corridor in China, it is a critical period for its operation and maintenance. Therefore, ensuring the safety of the integrated pipeline corridor and how to prevent and control the risks of operation and maintenance is a serious issue that needs urgent attention.

As engineering pipelines incorporated into the integrated pipeline corridor include power cables, communication cables, water supply, and drainage, heat and gas pipelines, etc., the operation and maintenance management team is complex, and it is difficult for the competent department to grasp the operation and maintenance status of the integrated pipeline corridor comprehensively. Therefore, this paper analyzes the relationship between the operation and maintenance risks of integrated pipeline corridors and studies the mechanism of risk paths in system operation and maintenance risks to put forward targeted references for the operation and maintenance management of integrated pipeline corridors.

\section{THE CURRENT STATUS OF INTEGRATED PIPELINE CORRIDOR RISK RESEARCH}

Integrated pipeline corridor operation and maintenance risk assessment is the main aspect of integrated pipeline corridor operation and maintenance management and is also the basis for preventing and controlling accidents in integrated pipeline corridors. The research on the risk assessment method of integrated pipeline corridor operation and maintenance mainly includes two aspects: qualitative and quantitative. Among them, qualitative risk assessment is carried out based on the index system of expert judgment and therefore is potentially subjective. Methods such as the analytic hierarchy process [4], fuzzy comprehensive judgment [5], and gray correlation analysis [6] are widely used in integrated pipeline corridor operation and maintenance risk assessment, which makes the determination of index weights and index assignment more scientific and objective. The quantitative risk assessment is mainly based on the physical model and calculation methods related to the integrated pipeline corridor, focusing on the quantitative risk assessment of the fire accident characteristics that have the greatest impact on the integrated pipeline corridor operation and maintenance risks. Among them, event tree analysis [7] and accident tree analysis [8] are used to analyze and predict the causes and probabilities of cable tunnel fires, and realize the quantitative expression of fire risk by calculating the consequences of fire; Bilal analyzed the consequences of pipeline fire and explosion accidents through the bow-tie analysis [9]; Mi combined the bow-tie analysis with Bayesian network to predict and analyze the failure probability of cable compartments in integrated pipeline corridors and explore the accident causation chain to provide a reference for fire risk analysis [10].

Although traditional risk assessment methods play an important role in identifying integrated pipeline corridor operation and maintenance risks and maintaining the operational safety of the corridor, the uncertainty and multi-source complexity of integrated management operation and maintenance risks require consideration of the impact of the interactions between the corridor risks. Therefore, the coupling model was used to evaluate the 
coupling relationship between risk factors of integrated pipeline corridors [11]; Wang analyzed the changing state of key influencing factors of integrated pipeline corridors under different hazard sensitivity values [12]; Feng explored to reveal the relationship between integrated pipeline corridor operation and maintenance safety and human factors [4]. However, the existing literature considers less the chain reaction and cyclic relationship between the risks of integrated pipeline corridor operation and maintenance, so the interdependent risks are managed as independent risks, which inevitably affects the effectiveness of risk response plan decisions.

In the field of project risk, Chen based on the improved neighborhood rough set theory to realize the whole process of integrated project risk management [13]. For the analysis and judgment of inter-risk relationships, Franck proposed a decision support system for project risks based on the combination of the dependency modeling approach of the design structure matrix (DSM) and the pairwise comparison principle of the analytic hierarchy process (AHP), which is used to realize the modeling and management of interactions among risks, thus facilitating managers to make decisions in response to risks [14].

System dynamics is a mathematical method for dynamic system analysis, originally developed by Professor Forrester at MIT in the late 1950s to focus on causal relationships and dynamic changes in systems while expressing multi-factor interactions [15]. Currently, system dynamics is being progressively applied to the evolution, simulation, and risk assessment of disaster systems [16, 17]. Since system dynamics has unique advantages in revealing the dynamic characteristics of system behavior, this study investigates the dynamic response process of system risk caused by various risk factors in the operation and maintenance management of integrated pipeline corridors over time based on system dynamics theory. Based on identifying the main risk factors in the operation and maintenance phase of integrated pipeline corridors, the risk factors affecting the safety of the corridor operation and maintenance are systematically classified. A system dynamics model of the risk is constructed to study the system morphological changes and analyze the risk paths through model simulation to provide help for the safe operation and maintenance management of the integrated pipeline corridor.

\section{RISK PATH ANALYSIS OF INTEGRATED PIPELINE CORRIDOR OPERATION AND MAINTENANCE}

In this paper, risk factors and their classification are determined from laws and regulations, standard specification documents [18, 19], previous literature related to integrated pipeline corridor operation and maintenance, and data from integrated pipeline corridor operation and maintenance experts. After modification and generalization, we finally get 5 types of Class I risks and 25 types of Class II risks as presented in Tab. 1.

\subsection{Comprehensive Pipeline Corridor Operation and Maintenance Risk Factors}

Before risk management, it is important to first determine the types of risk factors that affect the safety of integrated pipeline corridor operations and maintenance.
Risk factors are any events that increase the frequency or severity of the occurrence of risky incidents. Because there is still a certain lack of research on integrated pipeline corridor operation and maintenance risk, this paper not only combines the standard specification documents of integrated pipeline corridor operation and maintenance, but also extracts and analyzes the risk factors in public works tunnel literature and project operation risk management literature and obtains the integrated pipeline corridor operation and maintenance risk factors as shown in Tab. 1.

Table 1 Risk factor table of integrated pipeline corridor operation and maintenance

\begin{tabular}{|c|c|}
\hline & \\
\hline Level I risk & Level II risk \\
\hline \multirow{5}{*}{$\begin{array}{l}\text { Ontological security } \\
\quad \text { risk }(R 1)\end{array}$} & $\begin{array}{l}\text { Failure to regularly inspect and measure the } \\
\text { structure of the pipeline corridor }(r 11)\end{array}$ \\
\hline & Unreasonable design and construction $(r 12)$ \\
\hline & Structural cracks $(r 13)$ \\
\hline & Structural rust and corrosion $(r 14)$ \\
\hline & Uneven settlement $(r 15)$ \\
\hline \multirow{5}{*}{ Pipeline risk $(R 2)$} & Pipeline leakage $(r 21)$ \\
\hline & $\begin{array}{l}\text { Pipeline maintenance, inspection is not timely } \\
(r 22)\end{array}$ \\
\hline & Cable failure $(r 23)$ \\
\hline & Pipeline explosion, fire $(r 24)$ \\
\hline & Third party damage $(r 25)$ \\
\hline \multirow{4}{*}{$\begin{array}{l}\text { Environmental risk } \\
(R 3)\end{array}$} & Abnormal humidity, temperature $(r 31)$ \\
\hline & Toxic gas concentration abnormal $(r 32)$ \\
\hline & Natural disaster damage $(r 33)$ \\
\hline & Illegal invasion (r34) \\
\hline \multirow{4}{*}{ Equipment risk $(R 4)$} & $\begin{array}{l}\text { Ventilation, drainage and other equipment } \\
\text { abnormal }(r 41)\end{array}$ \\
\hline & $\begin{array}{l}\text { Risk monitoring and alarm equipment abnormal } \\
\qquad(r 42)\end{array}$ \\
\hline & Equipment is not linked $(r 43)$ \\
\hline & $\begin{array}{l}\text { Equipment maintenance and inspection is not } \\
\text { timely }(r 44)\end{array}$ \\
\hline \multirow{7}{*}{$\begin{array}{l}\text { Management risk } \\
(R 5)\end{array}$} & Management team level $(r 51)$ \\
\hline & Management system level (r52) \\
\hline & Information management level $(r 53)$ \\
\hline & Safety awareness level $(r 54)$ \\
\hline & Operation specification and technical level $(r 55)$ \\
\hline & Daily training and exercise level $(r 56)$ \\
\hline & Emergency management level $(r 57)$ \\
\hline
\end{tabular}

According to the content of the integrated pipeline corridor operation and maintenance safety hazard verification project, the risk is divided into the above five categories. In the level II risk, some of the risks are explained as follows: Unreasonable design and construction risks are potential hazards caused by irregularity with current standards and specifications. Uneven settlement risk includes deformation joints, branching mouth of the difference settlement. Third party damage risk is the risk of pipeline caused by city construction and third party road construction. Illegal invasion risk includes personnel illegal invasion caused by malicious arson, etc. Equipment is not linked risk includes cases where the fire door monitoring system does not link to close the fire door after detecting a fire.

\subsection{Risk Path Analysis of Integrated Pipeline Corridor Operation and Maintenance}

According to the categorization and organization of the risk factor table of integrated pipeline corridor operation and maintenance, all the level I risk factors are associated with the integrated risk of integrated pipeline corridor operation and maintenance, and all the level II risk factors 
are associated with the level I risk factors. Therefore, for these two aspects, Saaty's hierarchical analysis [20] was used to process the corresponding risk factors to obtain the weights of risk factors. The judgment matrix was formed by interviewing experts in the form of an online questionnaire and processing the collected questionnaire data.

\subsubsection{Analysis of the Relationship between Each Level of Risk}

According to the above method, a nine-level scale of risk intensity is used to first determine the weight vector, judgment matrix, and consistency test between the level I risk factors and the integrated risk of integrated pipeline corridor operation and maintenance, and between the level I risk factors and their corresponding level II risk factors.
Here, the relationship matrix between the level I risk factors and their corresponding level II risk factors is calculated as an example, and the calculation results are shown in Tab. 2.

When the consistency ratio is less than 0.1 , the degree of inconsistency is considered to be within the tolerable range and there is satisfactory consistency. However, the judgment matrix of $R 1$ in the first test did not pass the consistency test, and after analysis, it was learned that issues such as structural damage in the ontological safety risk were not the expertise of the operation and management staff, so the judgment matrix was adjusted by consulting experts in the construction of the integrated pipeline corridor and re-integrating their opinions. Tab. 2 shows the adjusted judgment matrix, and the consistency test shows that the judgment matrix obtained after the improvement successfully passed the consistency test and the optimization work is more successful.

Table 2 Level I risk and Level II risk weighting matrix

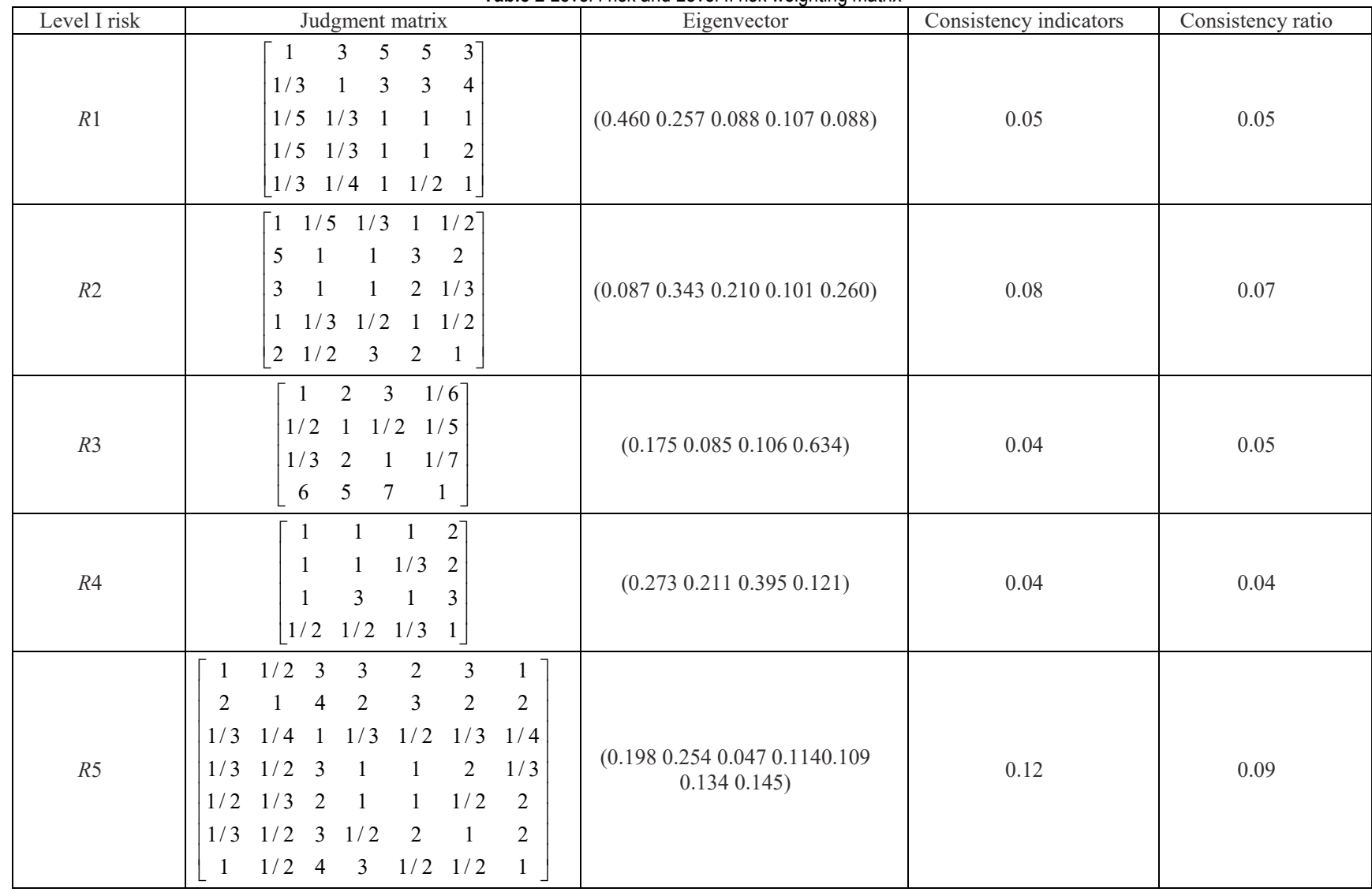

\subsubsection{Analysis of the Relationship between Level II Risks}

After determining the path relationships and weights between level I risk and the integrated risk of integrated pipeline corridor operation and maintenance and between level I risk and its corresponding level II risk, the next step is to identify the relevant path relationships among the level II risk factors and the path weights. For the analysis of the path relationships between the more complex level II risk factors, the relevant path relationships between the level II risk factors and the path coefficients were determined based on the improved DSM method combined with the study developed by Franck to obtain risk interaction strength values based on the AHP assessment method [14]. The results of determining the interaction relationships and intensities among the level II risk factors are shown in Tab. 3. Because Tab. 3 simultaneously represents the interaction relationship between level II risk factors, the constructed 0, 1 structure matrix is omitted

As can be seen in the table, the path relationship between risk factors of level II is complex, a risk factor may be caused by a variety of risk factors, and the consequences of different risk incidents caused by the same risk factor are not the same. The direction of the arrow in the table indicates the path relationship between risks, such as $r 11$ risk may cause $r 13, r 14$ and $r 15$ risk, and $r 11$ risk causes the most serious risk accident consequences $r 13$, with a weight of 0.310 , while $r 13$ risk is caused by $r 11, r 12, r 15, r 25$ and $r 33$ risk, of which $r 25$ risk is most likely to cause $r 13$ risk. 
Table 3 Pathway relationships and strengths among risk factors in Level II

\begin{tabular}{|c|c|c|c|c|c|c|c|c|c|c|c|c|c|c|c|c|c|c|c|c|c|c|c|c|c|}
\hline$\leftarrow$ & $r 11$ & $r 12$ & $r 13$ & $r 14$ & $r 15$ & $r 21$ & $r 22$ & $r 23$ & $r 24$ & $r 25$ & r31 & $r 32$ & $r 33$ & $r 34$ & $r 41$ & $r 42$ & $r 43$ & $r 44$ & $r 51$ & $r 52$ & $r 53$ & $r 54$ & $r 55$ & \begin{tabular}{|l|}
$r 56$ \\
\end{tabular} & $r 57$ \\
\hline$r 11$ & 0 & 0 & 0 & 0 & 0 & 0 & 0 & 0 & 0 & 0 & 0 & 0 & 0 & 0 & 0 & 0 & 0 & 0 & 0.461 & 0 & 0.326 & 0 & 0 & 0 & 0 \\
\hline$r 12$ & 0 & 0 & 0 & 0 & 0 & 0 & 0 & 0 & 0 & 0 & 0 & 0 & 0 & 0 & 0 & 0 & 0 & 0 & 0 & 0 & 0 & 0 & 0 & 0 & 0 \\
\hline$r 13$ & 0.310 & 0.264 & 0 & 0 & 0.253 & 0 & 0 & 0 & 0 & 0.420 & 0 & 0 & 0.219 & 0 & 0 & 0 & 0 & 0 & 0 & 0 & 0 & 0 & 0 & 0 & 0 \\
\hline$r 14$ & 0.287 & 0 & 0 & \begin{tabular}{|l}
0 \\
\end{tabular} & 0 & 0 & 0 & 0 & 0 & 0 & 0 & 0 & 0 & 0 & 0 & 0 & 0 & 0 & 0 & 0 & 0 & 0 & 0 & 0 & 0 \\
\hline$r 15$ & 0.287 & 0.231 & 0 & 0 & 0 & 0 & 0 & 0 & 0 & 0.451 & 0 & 0 & 0.278 & 0 & 0 & 0 & 0 & 0 & 0 & 0 & 0 & 0 & 0 & 0 & 0 \\
\hline$r 21$ & 0 & 0 & 0 & 0 & 0.500 & 0 & 0.433 & 0 & 0 & 0 & 0 & 0 & 0 & 0 & 0 & 0 & 0 & 0 & 0 & 0 & 0 & 0 & 0 & 0 & 0 \\
\hline$r 22$ & 0 & 0 & 0 & 0 & 0 & 0 & 0 & 0 & 0 & 0 & 0 & 0 & 0 & 0 & 0 & 0 & 0 & 0 & 0.362 & 0 & 0.256 & 0 & 0 & 0 & 0 \\
\hline$r 23$ & 0 & 0 & 0 & 0 & 0 & 0 & 0.408 & 0 & 0 & 0 & 0 & 0 & 0 & 0 & 0 & 0 & 0 & 0 & 0 & 0 & 0 & 0.333 & 0 & 0 & 0 \\
\hline$r 24$ & 0 & 0 & 0 & 0 & 0 & 0.303 & 0 & 0.619 & 0 & 0 & 0 & 0.246 & 0 & 0.310 & 0 & 0 & 0 & 0 & 0 & 0 & 0 & 0 & 0 & 0 & 0 \\
\hline$r 25$ & 0 & 0 & 0 & 0 & 0 & 0 & 0 & 0 & 0 & 0 & 0 & 0 & 0 & 0 & 0 & 0 & 0 & 0 & 0 & 0 & 0 & 0 & 0 & 0 & 0 \\
\hline$r 31$ & 0 & 0 & 0 & 0 & 0 & 0.305 & 0 & 0 & 0.560 & 0 & 0 & 0 & 0 & 0 & 0.354 & 0 & 0 & 0 & 0 & 0 & 0 & 0 & 0 & 0 & 0 \\
\hline$r 32$ & 0 & 0 & 0 & 0 & 0 & 0.281 & 0 & 0 & 0 & 0 & 0 & 0 & 0 & 0 & 0.408 & 0 & 0 & 0 & 0 & 0 & 0 & 0 & 0 & 0 & 0 \\
\hline$r 33$ & 0 & 0 & 0 & 0 & 0 & 0 & 0 & 0 & 0 & 0 & 0 & 0 & 0 & 0 & 0 & 0 & 0 & 0 & 0 & 0 & 0 & 0 & 0 & 0 & 0 \\
\hline$r 34$ & 0 & 0.218 & 0 & 0 & 0 & 0 & 0 & 0 & 0 & 0 & 0 & 0 & 0 & 0 & 0 & 0 & 0 & 0 & 0 & 0.385 & 0 & 0 & 0 & 0 & 0 \\
\hline$r 41$ & 0 & 0 & 0 & 0 & 0 & 0 & 0 & 0.259 & 0 & 0 & 0 & 0 & 0 & 0 & 0 & 0.293 & 0.177 & 0 & 0 & 0.176 & 0 & 0 & 0 & 0 & 0 \\
\hline$r 42$ & 0 & 0 & 0 & 0 & 0 & 0 & 0 & 0 & 0 & 0 & 0 & 0 & 0 & 0 & 0 & 0.558 & 0.232 & 0 & 0 & 0.389 & 0 & 0 & 0 & 0 & 0 \\
\hline$r 43$ & 0 & 0 & 0 & 0 & 0 & 0 & 0 & 0 & 0 & 0 & 0 & 0 & 0 & 0 & 0 & 0 & 0.550 & 0 & 0.239 & 0 & 0.169 & 0 & 0 & 0 & 0 \\
\hline$r 44$ & 0 & 0 & 0 & 0 & 0 & 0 & 0 & 0 & 0 & 0 & 0 & 0 & 0 & 0 & 0 & 0 & 0 & 0 & 0 & 0 & 0 & 0 & 0 & 0 & 0 \\
\hline$r 51$ & 0 & 0 & 0 & 0 & 0 & 0 & 0 & 0 & 0 & 0 & 0 & 0 & 0 & 0 & 0 & 0 & 0 & 0.638 & 0 & 0 & 0 & 0 & 0 & 0 & 0 \\
\hline$r 52$ & 0 & 0 & 0 & 0 & 0 & 0 & 0 & 0 & 0 & 0 & 0 & 0 & 0 & 0 & 0 & 0 & 0 & 0.158 & 0 & 0 & 0 & 0 & 0 & 0 & 0 \\
\hline$r 53$ & 0 & 0 & 0 & 0 & 0 & 0 & 0 & 0 & 0 & 0 & 0 & 0 & 0 & 0 & 0 & 0 & 0 & 0.262 & 0.436 & 0 & 0 & 0 & 0 & 0 & 0 \\
\hline$r 54$ & 0 & 0 & 0 & 0 & 0 & 0 & 0 & 0 & 0 & 0 & 0 & 0 & 0 & 0 & 0 & 0 & 0 & 0 & 0 & 0 & 0.547 & 0 & 0 & 0 & 0 \\
\hline$r 55$ & 0 & 0 & 0 & 0 & 0 & 0 & 0 & 0 & 0 & 0 & 0 & 0 & 0 & 0 & 0 & 0 & 0 & 0 & 0 & 0 & 0 & 0 & 0 & 0.293 & 0 \\
\hline$r 56$ & 0 & 0 & 0 & 0 & 0 & 0 & 0 & 0 & 0 & 0 & 0 & 0 & 0 & 0 & 0 & 0 & 0 & 0 & 0 & 0 & 0 & 0 & 0 & 0.354 & 0.707 \\
\hline$r 57$ & 0 & 0 & 0 & 0 & 0 & 0 & 0 & 0 & 0 & 0 & 0 & 0 & 0 & 0 & 0 & 0 & 0 & 0.568 & 0 & 0 & 0 & 0 & 0 & 0 & 0 \\
\hline
\end{tabular}

For the results in the table, further analysis can be done to explore the influence mechanism among the risks of integrated pipeline corridor operation and maintenance, such as the risk of pipeline explosion and fire accident (r24). These have a more serious impact on the safety of integrated pipeline corridor operation and maintenance and can be caused by pipeline leakage, cable failure and abnormal toxic gas concentration. Cable failure is more likely when there is third-party damage and can induce a fire accident in the corridor and cause further explosion hazard. And the pipeline leakage risk is slightly stronger than that of abnormal toxic gas concentration risk. As you can see from the consequences of pipeline leakage risk, the risk of abnormal toxic gas concentrations is only part of the consequences. Pipeline leakage risk can also cause the risk of abnormal humidity and temperature, so pipeline leak risk is even stronger.

\section{SYSTEM DYNAMICS MODEL OF INTEGRATED PIPELINE CORRIDOR OPERATION AND MAINTENANCE RISK}

This section is to construct a system dynamics model for the operation and maintenance risks of the integrated pipeline corridor. The risk relationship analysis in the previous section is converted to causality and the risk action intensity is converted to the weight coefficient of the risk path. On this basis, the dynamic changes of risk are analyzed by model simulation.

\subsection{Model Construction \\ 4.1.1 Boundaries and Research Hypotheses}

The integrated pipeline corridor operation and maintenance risk system presents complex and dynamic characteristics as time progresses. The system boundary as the research scope of the system aims to simplify the analysis model by identifying the key variables in the system. Therefore, with the integrated pipe corridor operation and maintenance risk as the research problem, the risk system is divided into five subsystems: ontological security risk, pipeline risk, environmental risk, equipment risk, and management risk according to the main contents of the operation and maintenance safety hazard verification project, and the following assumptions are made in combination with the actual situation of integrated pipeline corridor operation and maintenance.

Hypothesis 1: Only consider the impact of risk factors on the risk system in the operation and maintenance phase of the integrated pipeline corridor, without considering the risk factors in other phases of the corridor.

Hypothesis 2: Due to the difficulty and complexity of integrated pipeline corridor operation and maintenance management, risk control input is used as a means of regulation to play a role in controlling the risk of integrated pipeline corridor operation and maintenance.

Hypothesis 3: The causal relationship of the model is formed based on the results of the analysis of the relationship between the role of risk at all levels and the relationship between level II risks.

\subsubsection{Causality and Feedback Analysis}

Based on the above analysis, the Vensim-PLE software can be used to construct the causality diagram of integrated pipeline corridor operation and maintenance risk as shown in Fig. 1. From the figure, we can see that the complex causal relationship of integrated pipeline corridor operation and maintenance risk and that there are many feedback loops. At the same time, according to system dynamics theory, integrated pipeline corridor operation and maintenance risk is more regulated by negative feedback loops and the regulation of those loops in the system should be examined as effectively as possible. This paper therefore selects to represent a negative feedback loop involving each subsystem feedback loop.

The first negative feedback loop: integrated pipeline corridor operation and maintenance risk $\rightarrow+$ risk control input $\rightarrow-$ management risk $\rightarrow+$ integrated pipeline corridor operation and maintenance risk.

The second negative feedback loop: integrated 
pipeline corridor operation and maintenance risk $\rightarrow+$ risk control input $\rightarrow-$ management risk $\rightarrow-$ management system level $\rightarrow-$ failure to regularly test and measure the structure of the corridor $\rightarrow+$ ontological security risk $\rightarrow$ + integrated pipeline corridor operation and maintenance risk.

The third negative feedback loop: integrated pipeline corridor operation and maintenance risk $\rightarrow+$ risk control input $\rightarrow-$ management risk $\rightarrow-$ safety awareness level $\rightarrow$ - pipeline maintenance, inspection is not timely $\rightarrow+$ pipeline risk $\rightarrow+$ integrated pipeline corridor operation and maintenance risk.
The fourth negative feedback loop: integrated pipeline corridor operation and maintenance risk $\rightarrow+$ risk control input $\rightarrow$ - management risk $\rightarrow-$ safety awareness level $\rightarrow$ - pipeline maintenance, inspection is not timely $\rightarrow+$ cable failure $\rightarrow+$ pipeline explosion, fire $\rightarrow+$ abnormal humidity, temperature $\rightarrow+$ environmental risk $\rightarrow+$ integrated pipeline corridor operation and maintenance risk

The fifth negative feedback loop: integrated pipeline corridor operation and maintenance risk $\rightarrow+$ risk control input $\rightarrow-$ management risk $\rightarrow-$ information management level $\rightarrow-$ equipment not linked $\rightarrow+$ equipment risk $\rightarrow+$ integrated pipeline corridor operation and maintenance risk.

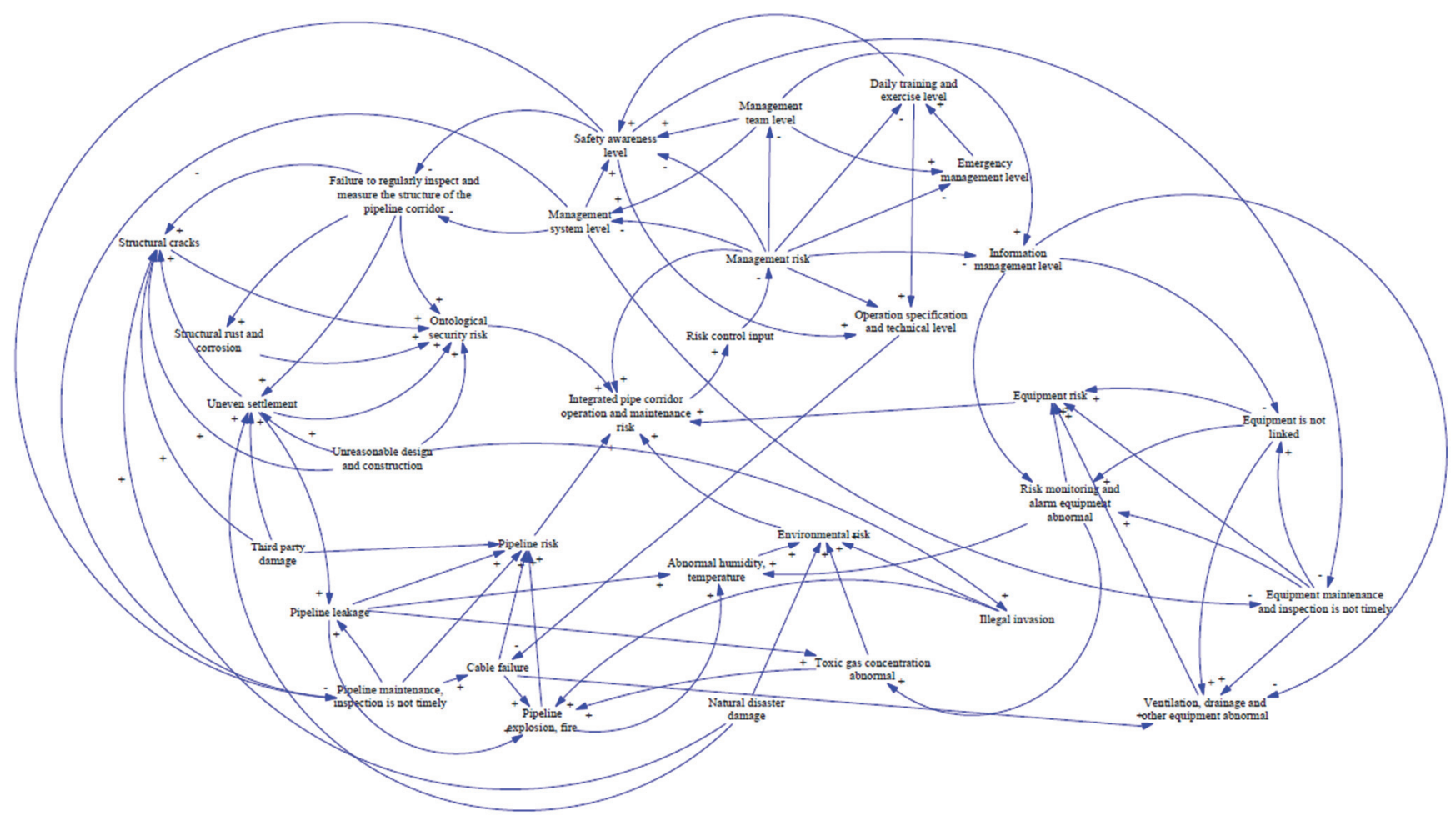

Figure 1 Integrated pipeline corridor operation and maintenance risk causality diagram

\subsubsection{Model Flow Diagram and Main Function Relationship Construction}

On the basis of the causality diagram, the level variables, rate variables, auxiliary variables, and constants are further extracted to construct the stock flow diagram of the target system as shown in Fig. 2.

Further, all the variables were summarized in conjunction with their meanings, and the results of the main variables are shown in Tab. 4 .

According to the main variables in Tab. 4, the INTEG function, TABLE function, SMOOTH function and DELAYI function are used comprehensively to construct the system of functional equations of the integrated pipeline corridor risk SD model containing state variables, rate variables, auxiliary variables and constant equations, and the path coefficients between risks are assigned with the results of the interaction strength between risks in the above sections when constructing the system of functions, as shown in Tab. 5.
Table 4 The main variables of the integrated pipeline corridor operation and maintenance risk system table

\begin{tabular}{|c|c|c|}
\hline $\begin{array}{l}\text { Variable } \\
\text { type }\end{array}$ & Variable name & Symbol \\
\hline \multirow{6}{*}{$\begin{array}{c}\text { Horizontal } \\
\text { variables }\end{array}$} & $\begin{array}{l}\text { Integrated pipe corridor operation } \\
\text { and maintenance risk }\end{array}$ & $R$ \\
\hline & Ontological security risk & $R 1$ \\
\hline & Pipeline risk & $R 2$ \\
\hline & Environmental risk & $R 3$ \\
\hline & Equipment risk & $R 4$ \\
\hline & Management risk & $R 5$ \\
\hline \multirow{8}{*}{$\begin{array}{c}\text { Rate } \\
\text { Variables }\end{array}$} & Ontological security risk increment & $f 1$ \\
\hline & Pipeline risk increment & $f 2$ \\
\hline & Environmental risk increment & $f 3$ \\
\hline & Equipment risk increment & $f 4$ \\
\hline & Management risk increment & $f 5$ \\
\hline & Management risk reduction & $f 6$ \\
\hline & $\begin{array}{l}\text { Integrated pipe corridor operation } \\
\text { and maintenance risk increment }\end{array}$ & FLOW1 \\
\hline & $\begin{array}{l}\text { Integrated pipe corridor operation } \\
\text { and maintenance risk reduction }\end{array}$ & FLOW2 \\
\hline $\begin{array}{l}\text { Auxiliary } \\
\text { variables }\end{array}$ & risk control input & $P$ \\
\hline \multirow{4}{*}{ Constant } & $\begin{array}{l}\text { Unreasonable design and } \\
\text { construction }\end{array}$ & $r 12$ \\
\hline & Third-party damage & $r 25$ \\
\hline & Natural disaster damage & $r 33$ \\
\hline & Management team level & $r 51$ \\
\hline
\end{tabular}




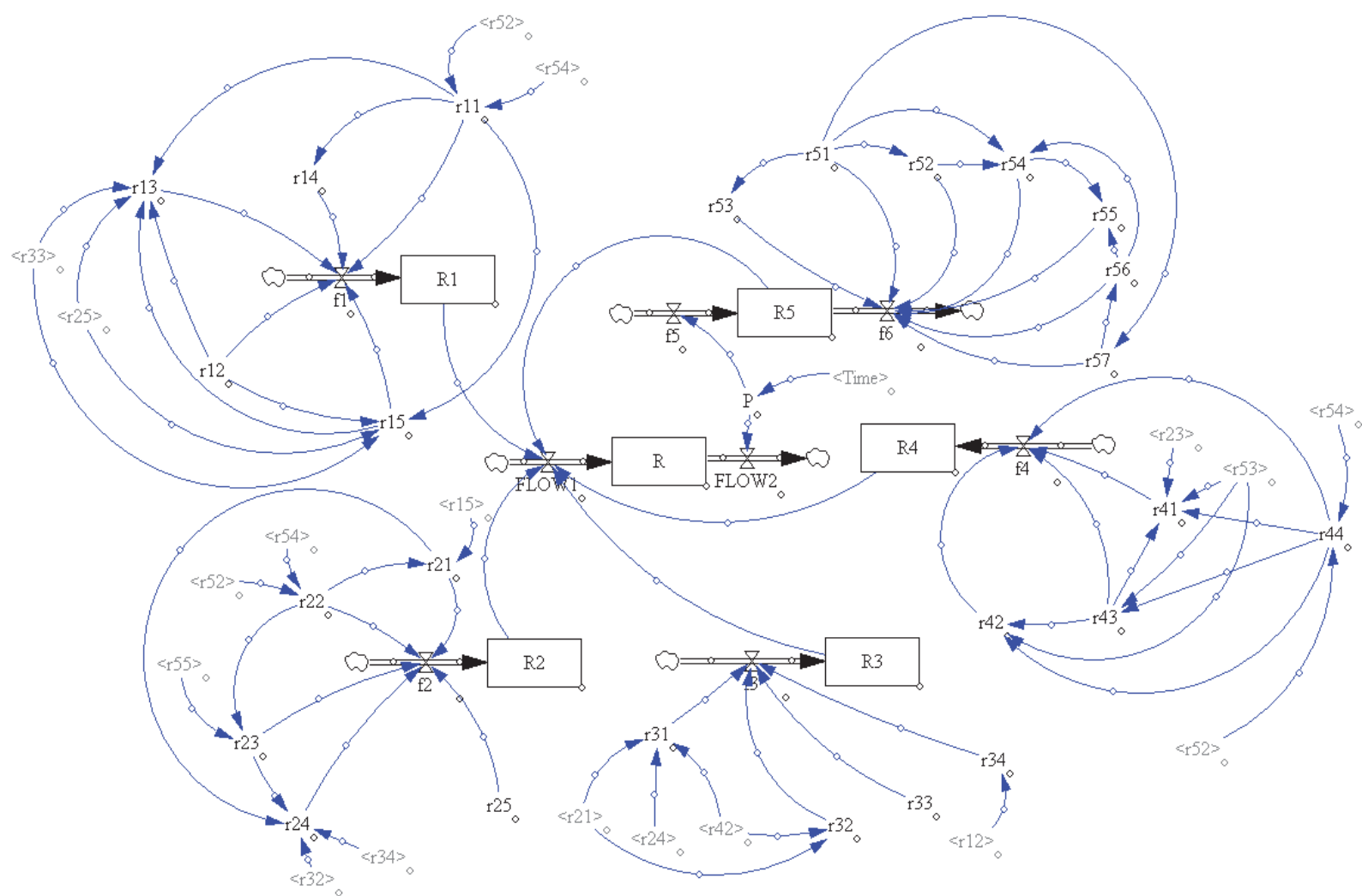

Figure 2 Integrated pipeline corridor operation and maintenance risk stock flow chart

Table 5 Functional equations for the SD model

\begin{tabular}{|c|c|}
\hline Symbol & Function equation \\
\hline$R$ & INTEG $(F L O W 1-F L O W 2,5)$ \\
\hline$R 1$ & INTEG $(\operatorname{SMOOTH}(f 1,5), 0)$ \\
\hline$R 2$ & $\operatorname{INTEG}(\operatorname{SMOOTH}(f 2,5), 0)$ \\
\hline$R 3$ & $\operatorname{INTEG}(\operatorname{SMOOTH}(f 3,5), 0)$ \\
\hline$R 4$ & INTEG $(\operatorname{SMOOTH}(f 4,5), 0)$ \\
\hline$R 5$ & $\operatorname{INTEG}(\operatorname{SMOOTH}(f 5-f 6,10), 0)$ \\
\hline$f 1$ & $D E L A Y 1 I\left(\begin{array}{l}0.46 * r 11+0.257 * r 12+0.088 * \\
r 13+0.107 * r 14+0.088 * r 15,3,0\end{array}\right)$ \\
\hline$f 2$ & $D E L A Y 1 I\left(\begin{array}{l}0.087 * r 21+0.343 * r 22+0.21 * \\
r 23+0.101 * r 24+0.26 * r 25,1,0\end{array}\right)$ \\
\hline$f 3$ & $D E L A Y 1 I\left(\begin{array}{l}0.175 * r 31+0.085 * r 32+0.106 * \\
r 33+0.634 * r 34,1,0\end{array}\right)$ \\
\hline$f 4$ & $D E L A Y 1 I\left(\begin{array}{l}0.273 * r 41+0.211 * r 42+0.395 * \\
r 43+0.121 * r 44,3,0\end{array}\right)$ \\
\hline$f 5$ & $1-P$ \\
\hline$f 6$ & $D E L A Y 1 I\left(\begin{array}{l}0.198 * r 51+0.254 * r 52+0.047 * \\
r 53+0.114 * r 54+0.109 * r 55+ \\
0.134 * r 56+0.145 * r 57,5,0\end{array}\right)$ \\
\hline FLOW1 & SMOOTH $\left(\right.$ DELAY3 $\left(\left(\begin{array}{l}0.569 * R 1+0.057 * \\
R 2+0.091 * R 3+ \\
0.079 * R 4+0.203 * R 5\end{array}\right) / 5,3\right.$ \\
\hline FLOW2 & $P$ \\
\hline$P$ & WITHLOOKUP $\left(\right.$ Time,$\left.\left(\begin{array}{l}{[(0,0)-(25,10)],(1,1),(1.25,0.8),} \\
(1.5,0.67),(1.75,0.57),(2,0.5), \\
(4,0.25),(5,0.2),(8,0.125),(10,0.1)\end{array}\right)\right)$ \\
\hline$r 12$ & 0.257 \\
\hline$r 25$ & 0.260 \\
\hline$r 33$ & 0.106 \\
\hline$r 51$ & 0.198 \\
\hline
\end{tabular}

\subsection{Simulation Model Experiments 4.2.1 Model Parameter Setting}

Because the relationship and intensity of the action between risks in the system dynamics model in this paper is determined by the path relationship and path weight coefficients in the previous chapter, the equations are set to dimensionless units. The risk of each subsystem is not yet presented in the early stage, so the risk value increases with time from 0 to the maximum value of 1 . The risk of integrated pipeline corridor operation and maintenance is limited by the fact that the operation and maintenance work has not yet been carried out and the risk control input has not yet played a role, resulting in the highest risk in the early stage of integrated pipeline corridor operation and maintenance, which is accumulated by the sum of the risk of each subsystem from 5 with time.

Risk control inputs according to the actual operation and maintenance in the same proportion within the interval $[0,1]$ for conversion. Due to the complex management of integrated pipeline corridor operation and maintenance at the beginning, the risk control input is the largest at this time. With the increase of time management tends to be stable, risk control input also gradually reduced to a certain input value no longer change. Therefore, this article refers to the risk control input in actual work to set the table function, and the minimum risk control input is 0.1 .

One year is used as the time for the SD model of integrated pipeline corridor operation and maintenance risk, the initial time is 0 , the end time is 12 , the time unit is month, and TIME STEP is 1.

\subsubsection{Model Validity Test}

The validity of the simulation model is tested by 
observing whether the dynamic evolution of the model is consistent with the behavior pattern of the real system, and by determining whether the results obtained by using the model to simulate the real system are correct and reliable. In this paper, we use Vensim-PLE software to check the accuracy and validity of the model by running a runtime test.

Further, the reasonableness of the model is judged based on the simulation results, and whether there is a large difference in the change trend of the study object at different time steps is simulated. In this paper, we simulated the change trend of integrated pipeline corridor operation and maintenance risk for time step $0.5,1$ and 1.5 respectively, as shown in Fig. 3.

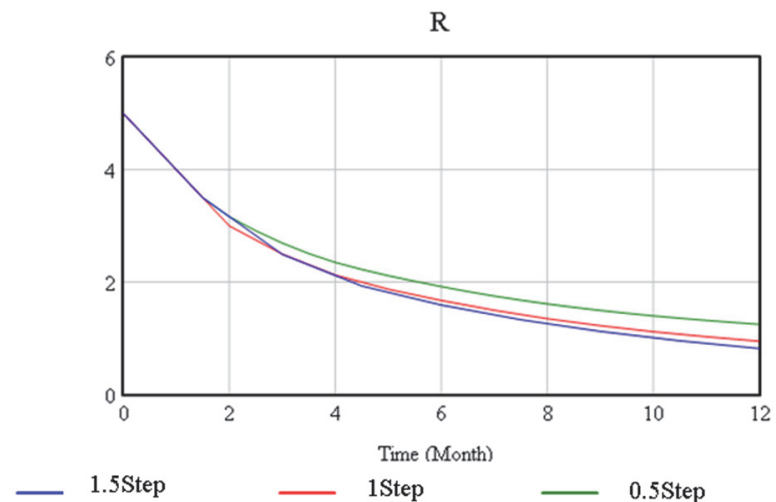

Figure 3 Simulation results of integrated pipeline corridor operation and maintenance risks under different step

The figure shows that the change trend of the integrated pipeline corridor operation and maintenance risk under different time steps is approximately the same. Integrated pipeline corridor operation and maintenance risk with the operation and maintenance work, the risk value decreases rapidly, with the stability of the operation and maintenance management and risk control input, integrated pipeline corridor operation and maintenance risk changes tend to stabilize and converge to a certain level value. But in practice, the impact of each risk factor cannot be completely eliminated, the system operation and maintenance risk value is not zero. This is consistent with the actual. In summary, it shows that the model is stable and feasible, and further simulation analysis can be conducted.

\subsubsection{Simulation Analysis}

The analysis of the flow chart of the simulation model of integrated pipeline corridor operation and maintenance risk shows that within the boundary of the system of limited scope, the whole system consists of four basic parameters: unreasonable design and construction $r 12$, third party damage $r 25$, natural disaster damage $r 33$, and management team level $r 51$. The remaining risks result from a dynamic variable which exists within the system. This can be understood as the remaining risk factors are based on these four risks and form a complete system through the cyclic action of risks. Therefore, the sensitivity analysis and testing of the four parameters constituting the system can be carried out to determine the degree of influence of these basic risks on the risks of each subsystem according to the simulation model of integrated pipeline corridor operation and maintenance risks constructed in this paper. By changing the increments of the four parameters separately, the results of the influence of the parameters on the risk of each subsystem are viewed, and the simulation results of $r 51$ as an example are shown in Figs. 4 to 8 .

R1

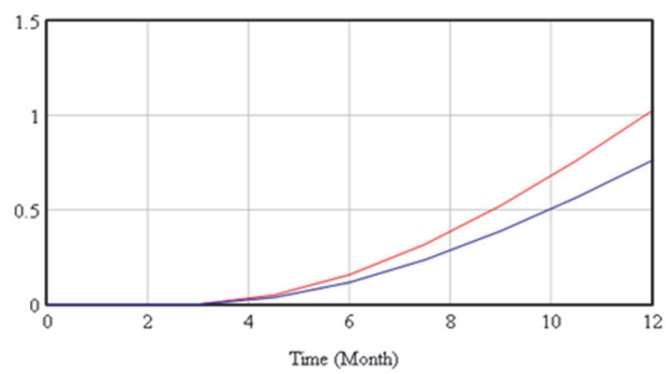
$\mathrm{r} 51$ increment 0.2 , other increments unchanged

Figure 4 Simulation results of the effect of $r 51$ on the risk of $R 1$ subsystem

R2

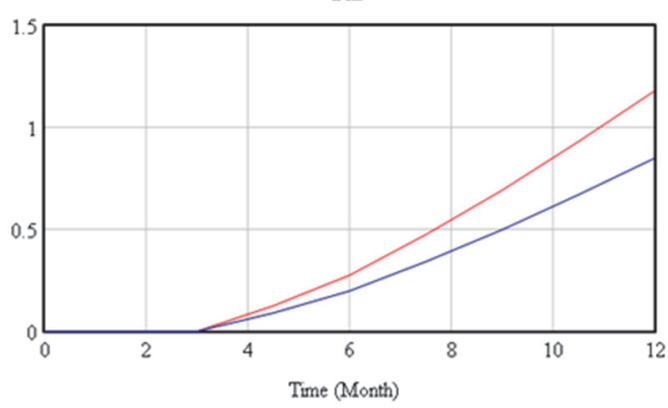

r 51 increment 0.2 , other increments unchanged Initial results

Figure 5 Simulation results of the effect of $r 51$ on the risk of $R 2$ subsystem

R3

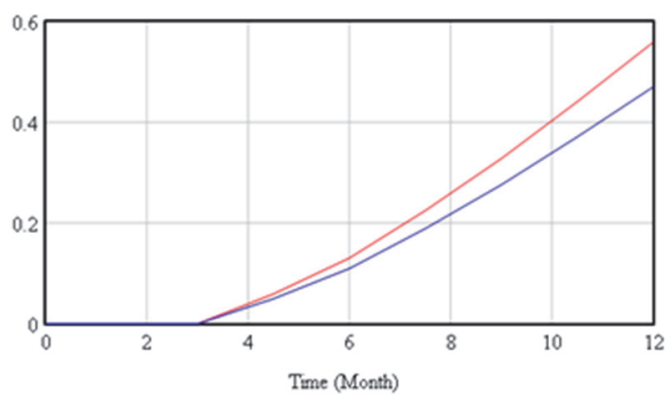

$\mathrm{r} 51$ increment 0.2 , other increments unchanged Initial results

Figure 6 Simulation results of the effect of $r 51$ on the risk of $R 3$ subsystem

R4

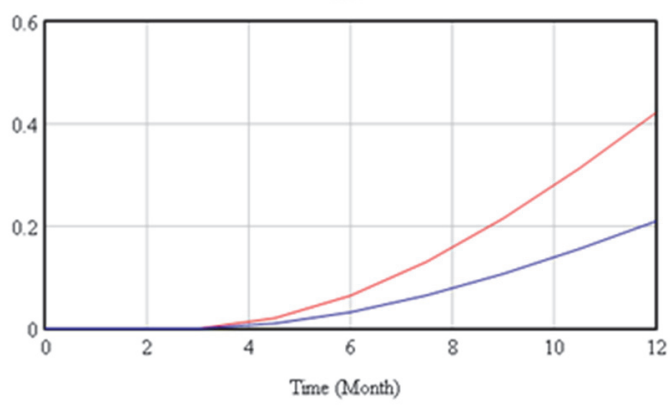

r51 increment 0.2 , other increments unchanged Initial results

Figure 7 Simulation results of the effect of $r 51$ on the risk of $R 4$ subsystem 
R5

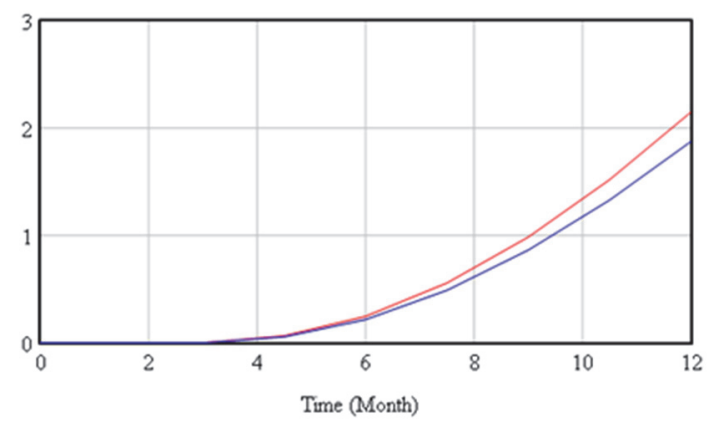

r51 increment 0.2 , other increments unchanged

Initial results

Figure 8 Simulation results of the effect of $r 51$ on the risk of $R 5$ subsystem

The simulation results show that under the influence of management team level $r 51$, the risk level of each subsystem changes, but the greatest influence is on the subsystem of equipment risk $R 4$, which changes by more than $50 \%$, and the subsystems of ontological security risk $R 1$, pipeline risk $R 2$ and environmental risk $R 3$ change less, while the subsystem of management risk $R 5$ is relatively insensitive. According to the simulation results, the problem of accidents caused by equipment risks can be indirectly reduced by improving the level of the integrated pipeline corridor operation and maintenance management team, and the purpose of regulating equipment risks can be achieved. In addition to the sensitivity analysis of the other three risks, the simulation results show that the risk $r 12$ only has a certain impact on the ontological security risk $R 1$ subsystem and environmental risk $R 3$ subsystem of the corridor to which it belongs, while it has almost no impact on the pipeline risk $R 2$, equipment risk $R 4$ and management risk $R 5$; risk $r 25$ has a greater impact on the pipeline risk $R 2$ and has a greater impact on the ontological security risk $R 1$ and environmental risk $R 3$ have a small impact; risk $r 33$ has almost no impact on equipment risk $R 4$ and management risk $R 5$.

$\mathrm{R}$

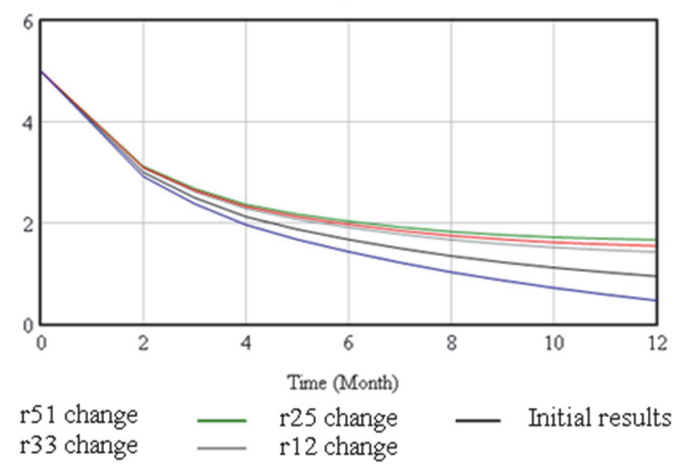

Figure 9 Simulation results of integrated pipeline corridor operation and maintenance risk $R$ under incremental change

The results of further analysis of the impact on the integrated pipeline corridor operation and maintenance risk under incremental changes are shown in Fig. 9. The simulation results show that the improvement of the management team level can significantly reduce integrated pipeline corridor operation and maintenance risk. Unreasonable design and construction, third-party damage and natural disaster damage risk increase will significantly affect integrated pipeline corridor operation and maintenance risk. The impact of third-party damage risk is more serious. Therefore, the process of integrated pipeline corridor operation and maintenance management needs not only to pay attention to the hazards of risks such as thirdparty damage but also to improving the level of the management team to achieve the purpose of indirectly regulating other risks and reducing the risk of integrated pipeline corridor operation and maintenance.

By extending the simulation time to 18 months, the integrated pipeline corridor operation and maintenance risk increases from the stable value. This is because with the increase of integrated pipeline corridor operation and maintenance time, operation and maintenance work tends to be stable, but the degree of aging of various equipment in the integrated pipeline corridor increases with time. In addition, management system, personnel training and other factors are not improved simultaneously, so the risk control input that has gradually tended to a certain level cannot completely offset the operation and maintenance risk brought about by such situations. The simulation is shown in Fig. 10.

$\mathrm{R}$

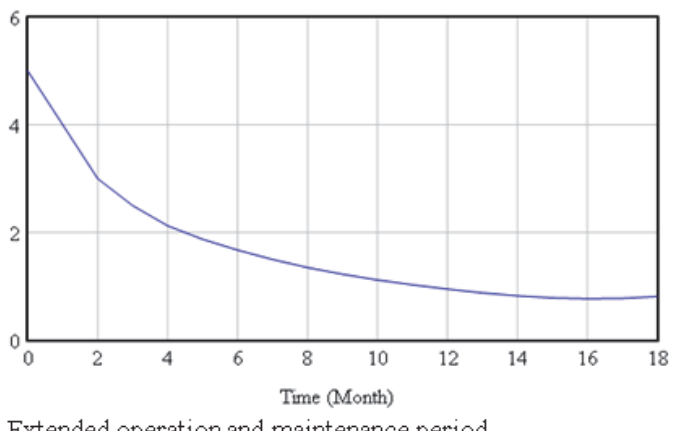

Extended operation and maintenance period

Figure 10 Simulation changes of integrated pipeline corridor operation and maintenance risk with extended operation and maintenance period

R

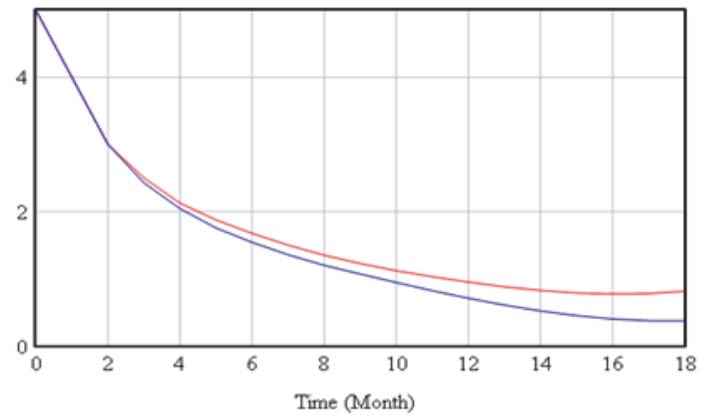

Increase risk control input —_ Extended O\&M period

Figure 11 Simulated changes in integrated pipeline corridor operation and maintenance risks after extending the period and increasing risk control input

In the face of the above-mentioned situation, the risk control input is increased to offset the changes in the derived risks caused by the increase in operation and maintenance time. The simulation shows that by increasing the proportion of risk control input, the above risk situation can be effectively reduced, and the purpose of reducing the risk of integrated pipeline corridor operation and maintenance can be achieved. The simulation results are shown in Fig. 11. 


\section{CONCLUSION AND DISCUSSION}

In the period of the expansion of integrated pipeline corridors and their operation and maintenance in China within the background of digitalization and increased socio-economic complexity, how to carry out effective risk management and how to predict and respond to the risks after the construction of integrated pipeline corridors effectively and in advance has become a key issue in future management. In this paper, based on existing research results in the field of integrated pipeline corridors, risk is analyzed by constructing a table of risk factors in integrated pipeline corridor operation and maintenance and using a combination of mathematical analysis and simulation modeling. The above research supplies the following insights into integrated pipeline corridor operation and maintenance risk management:

(1) Through existing literature research, a list of risk factors in integrated pipeline corridor operation and maintenance is formed. This risk list can provide reference for the subsequent study of integrated pipeline corridor operation and maintenance risks.

(2) Through the study of the relationship between risks and the strength of that relationship, the mechanism of action between integrated pipeline corridor operation and maintenance risks is analyzed and the causal relationship between different risks is elaborated, which helps operation and maintenance managers to better understand and predict the causes and consequences of risks. For major risks such as pipeline explosion and fire, risk can be reduced, and the safety of operation and maintenance can be improved by controlling its critical factors or eliminating its risk path.

(3) The analysis conducted by simulation models shows the trend of dynamic change of integrated pipeline corridor operation and maintenance risks over time. The focus should be on risk sources such as at the team management level; safe operation and maintenance should be improved by forming an efficient operation and maintenance management team and strengthening the synergy of each member. In addition, we should focus on increasing the risk control input to cope with the derivative risks brought by the growth of the integrated pipeline corridor over time. In this paper, risk control input is used as a control tool to reduce the risk, and in the actual work, risk control input can be specifically converted into the update of equipment, the construction of intelligent operation and maintenance platforms, and the standardization and integration of management and other operation and maintenance work content.

Although this paper investigates the dynamic changes in the operation and maintenance risks of integrated pipeline corridors, the attribution of risk factors in specific pipeline corridors has been omitted due to the differences in regions. The research in this paper focuses on the overall analysis and control of risk trends, but there is a certain degree of subjectivity in the measurement of risk values. Therefore, how to combine the monitoring data of integrated pipeline corridor operation and maintenance and use machine learning-related algorithms to make the measurement of integrated pipeline corridor risk values more objective needs further research in the future.

\section{Acknowledgements}

This work was supported by Beijing Social Science Foundation under Grant 19JDGLA002, 18JDGLA018, 21JCB093, 21JCC092, MOE (Ministry of Education in China) Project of Humanities and Social Sciences under Grant 19YJC630043, the National Natural Science Foundation of China under Grant J1824031 and was partially supported by Beijing Logistics Informatics Research Base.

\section{REFERENCES}

[1] Qian, Q. H. (2017). Construction of urban underground integrated pipeline corridor, transforming urban development. Tunnel Construction, 37(6), 647-654.

[2] Wang, T., Tan, L., Xie, S., \& Ma, B. (2018). Development and applications of common utility tunnels in China. Tunneling and Underground Space Technology, 76, 92-106. https://doi.org/10.1016/j.tust.2018.03.006

[3] Guo, J. Q., Qian, Y., Wang, Z. Z., Dun, Z. L., \& Liu, X. L. (2019). Research on common operation and maintenance disasters and countermeasures of urban underground integrated pipe corridors. Disaster Science, 34(01), 27-33. https://doi.org/CNKI:SUN:ZHXU.0.2019-01-006

[4] Feng, D. K., Sun, Z. S., Wu, C. L., \& Zhang, Z. Y. (2019). Analysis and Countermeasures of Threats of Human Damage in Urban Underground Integrated Pipe Corridors. Journal of Underground Space and Engineering, 15(S2), 513-521. https://doi.org/CNKI:SUN:BASE.0.2019-S2-003

[5] Chai, K. \& Liu, X. (2020). Multi-hazard coupled prediction model for integrated pipe corridors based on fuzzy cluster analysis. Disaster Science, 35(04), 206-209. https://doi.org/CNKI:SUN:ZHXU.0.2020-04-038

[6] Long, D. B., Zhao, Y. J., \& Yang, C. (2021). Dynamic risk assessment of integrated corridor operation and maintenance driven by monitoring alarm data. Journal of Safety and Environment, 1-11. https://doi.org/10.13637/j.issn.1009-6094.2021.0429

[7] Fang, H. Q. (2019). Urban power cable tunnel fire risk assessment study. Master Thesis, Beijing: University of Science and Technology of China.

[8] Shen, Y. (2016). Fire and explosion hazard analysis and evaluation study of thermal power plants, Master Thesis, Xi'an: Xi'an University of Architecture and Technology.

[9] Bilal, Z., Mohammed, K., \& Brahim, H. (2016). Bayesian network and bow tie to analyze the risk of fire and explosion of pipelines. Process Safety Progress, 36(2), 202-212. https://doi.org/10.1002/prs.11860

[10] Mi, H. F. \& Zhang, X. M. (2021). Probabilistic analysis method of cable compartment fire in urban underground integrated pipeline corridor. Journal of Chinese Safety Science, 31(01), 165-172. https://doi.org/10.16265/j.cnki.issn1003-3033.2021.01.024

[11] Wang, S. H., Zhang, Z., Hou, W. S., \&Wang, F. L. (2018). Multi-hazard coupled disaster risk evaluation method for integrated pipeline corridors. Journal of Northeastern University (Natural Science Edition), 39(06), 902-906. https://doi.org/CNKI:SUN:DBDX.0.2018-06-028

[12] Wang, W., Zhang, X. X., Zhu, B. Q., \& Teng, J. Y. (2020). Analysis of Key Influencing Factors for Intelligent Operation and Maintenance of Integrated Pipe Corridors. Laboratory Research and Exploration, 39(11), 30-34. https://doi.org/CNKI:SUN:SYSY.0.2020-11-007

[13] Chen, W., Wang, J., \& Wang, C. (2020). Study of Risk Evaluation for Complex Projects under BIM and IPD Collaborative Pattern Based on Neighborhood Rough Sets. 
Tehnicki vjesnik - Technical Gazette, 27(2), 444-449. https://doi.org/10.17559/TV-20191022112633

[14] Fang, C. \& Marle, F. (2012). A simulation-based risk network model for decision support in project risk management. Decision Support Systems, 52(3), 635-644. https://doi.org/10.1016/j.dss.2011.10.021

[15] Forrester, J. W. (2012). Industrial Dynamics: A Major Breakthrough for Decision Makers. The Roots of Logistics, 141-172. https://doi.org/10.1007/978-3-642-27922-5_13

[16] Wang, Y. F., Li, B., Qin, T., \& Zhang, B. (2018). Probability prediction and cost benefit analysis based on system dynamics. Process Safety and Environmental Protection, 114, 271-278. https://doi.org/10.1016/j.psep.2018.01.002

[17] Yan, W., Wang, J., \& Jiang, J. (2016). Subway Fire Cause Analysis Model Based on System Dynamics: A Preliminary Model Framework. Procedia Engineering, 135, 431-438. https://doi.org/10.1016/j.proeng.2016.01.152

[18] GB50838. (2015). Urban integrated pipeline corridor engineering technology standards. Beijing

[19] GB51354. (2019). Urban underground integrated pipeline corridor operation and maintenance and safety technical standards. Beijing

[20] Saaty, T. L. (2003). Decision-making with the AHP: Why is the principal eigenvector necessary? European Journal of Operational Research, 145(1), 85-91. https://doi.org/10.1016/s0377-2217(02)00227-8

\section{Contact information:}

\section{Weixin FENG, Master}

(Corresponding author)

Beijing Jiaotong University,

No. 3, Shangyuan Village, Haidian District, Beijing, China

E-mail: 19120564@bjtu.edu.cn

DAN CHANG, Professor

Beijing Jiaotong University,

No. 3, Shangyuan Village, Haidian District, Beijing, China

E-mail: changdan@bjtu.edu.cn

\section{Daqing GONG, Associate Professor}

Beijing Jiaotong University,

No. 3, Shangyuan Village, Haidian District, Beijing, China

E-mail: dqgong@bjtu.edu.cn

\section{Jiren SHEN}

Beijing Capital Agribusiness \& Food Group,

Beijing 100000, China 